\title{
Beyond particle physics
}

\author{
Topological semimetals give access to new quantum phenomena - for example, massless fermions \\ have not been observed as elementary particles, yet they can be realized in the form of quasiparticles in \\ these materials - and could allow the development of robust quantum devices.
}

Since the prediction and experimental realization of topological insulators a decade ago, topological materials have become one of the mainstream research topics in condensed matter physics. The conductive properties of topological matter depend on the topological properties of the bulk electron wave functions, and are therefore a paradigmatic example of quantum materials - those in which quantum properties are observable at the macroscopic scale. Topological insulators, in particular, are insulators in their bulk, but they have conductive surface states due to spin-orbit coupling and time-reversal symmetry, or due to crystalline symmetries ${ }^{1,2}$. Although these materials still provide a fertile ground for new developments, in the past couple of years the main focus of research has moved towards topological semimetals.

In 1928, Paul Dirac published an equation for the description of the quantum properties of relativistic fermions. Soon after, Hermann Weyl noticed that, assuming the existence of particles with no mass, 'Weyl fermions', the equation could be split into a system of two with non-trivial solutions distinguished by chirality, a feature that implies that spin and momentum are correlated ${ }^{3}$. These massless fermions have not been detected in nature so $\mathrm{far}^{3}$. In 2011, however, some theoretical studies predicted their possible realization in the form of quasiparticles in material systems characterized by conduction and valence bands locally forming Dirac cones similar to those found in graphene (reviewed by Anton A. Burkov on page 1145 of this issue) and proximal to the Fermi energy level. In these materials, called Weyl semimetals, the touching points (or Weyl nodes) are topologically protected due to their crystal structure asymmetries or their magnetic nature. Weyl semimetals are particularly interesting because they reflect several quantum anomalies arising from the chiral properties of the Weyl fermions - with no equivalent in quantum particle physics and show unique transport, optical and thermoelectric responses. For instance, the electronic structure leads to the formation of surface spin-polarized states in the form of arcs in momentum space (so called Fermi arcs) (Fig. 1).

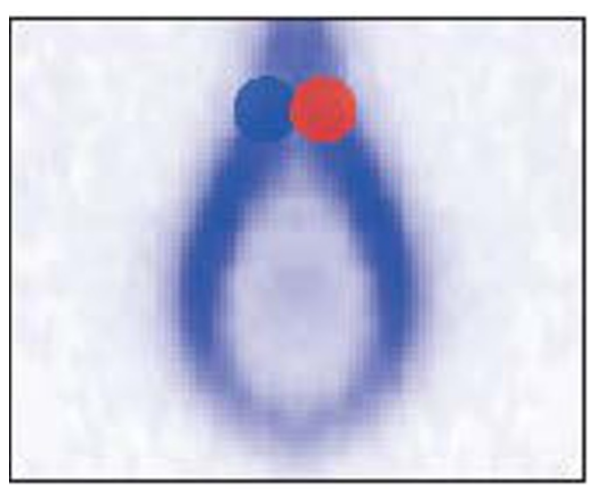

Figure 1| Momentum space measurement obtained through ARPES showing a Fermi arc (blue area) at the surface of NbP. It connects the surface projections of two Weyl nodes with different chirality (represented by the red and blue dots).

Figure adapted from ref. 4, Nature Publishing Group.

In his Commentary, Burkov discusses the theoretical basis for the existence of topological semimetals and their unique properties. Weyl semimetals can in fact be classified depending on the form in which the conduction and valence bands touch each other. They can form Dirac cones that are tilted (type II) or not (type I), which leads to distinct properties. In addition, when four different bands degenerate locally in a so-called Dirac point, another family of topological materials named Dirac semimetals is formed (see, for example, ref. 4).

In 2015, non-centrosymmetric transitionmetal monophosphides were predicted to be Weyl type I semimetals, and a few months later TaAs and $\mathrm{NbAs}$ were confirmed as such $^{5-8}$. More Weyl semimetals within that family of compounds have been reported since then, and ways to fine-tune their topological properties have been demonstrated ${ }^{9}$. In spite of these advances, the number of Weyl semimetals is still limited, and they typically show a combination of topological and non-topological properties. On page 1161 of this issue, Nai Phuan Ong and colleagues report the possibility of inducing the formation of Weyl points in halfHeusler alloys by applying magnetic fields, which may potentially lead to a large increase in the number of Weyl semimetals available.
Type II Weyl semimetals were also predicted in $2015^{10}$. On page 1155 , Lunan Huang and colleagues now report spectroscopic evidence for a type II Weyl semimetallic state in $\mathrm{MoTe}_{2}$.

Such short timescales from theoretical predictions to experimental realizations have only been possible due to the collaboration and competition among different groups worldwide, and by no means indicate elementary experimental work following the theoretical findings. Angle resolve photoelectron emission spectroscopy (ARPES) and transport measurements, are currently the workhorse characterization techniques. On page 1140 of this issue, M. Zahid Hasan and colleagues explain the experimental challenges to obtaining and characterizing Weyl semimetals, and more importantly, to correctly interpret ARPES data.

The excitement surrounding topological semimetals is not only due to the possibility of studying new quantum phenomena, or to the realization of new exotic phases of matter. It also springs from the promise of developing quantum devices working at relatively high temperature, as the topological protection makes their quantum properties robust against perturbations. In their Commentary, M. Zahid Hasan and collaborators also suggest strategies for controlling these properties by optical and electrical means, aiming to take full advantage of them in devices.

Although promising, topological semimetals face a major hurdle towards applicability. As Burkov points out, new materials should be found where the topological properties are accessible and dominate other possible trivial contributions. Obtaining such a material is, hopefully, just a matter of time.

\footnotetext{
References

1. Hasan, M. Z. \& Kane, C. L. Rev. Mod. Phys. 82, 3045 (2010).

2. X.-L. Qi and S. -C. Zhang, Rev. Mod. Phys. 83, 1057 (2011).

3. Ciudad, D. Nat. Mater. 14, 863 (2015).

4. Liu, Z. K. Nat. Mater. 13, 677-681 (2014).

5. Huang, S. M. et al. Nat. Commun. 6, 7373 (2015).

6. Weng, H. et al. Phys. Rev. X 5, 011029 (2015).

7. Xu, S. Y. et al. Science 349, 613-617 (2015).

8. Lv, B. Q. et al. Phys. Rev. X 5, 031013 (2015).

9. Liu, Z. K. et al. Nat. Mater. 15, 27-31, (2016).

10. Soluyanov, A. A. et al., Nature 527, 495-498 (2015).
} 